\title{
Association of traditional Chinese medicine therapy and the risk of dementia in patients with hypertension: a nationwide population-based cohort study
}

Kuen-Hau Chen ${ }^{1}$, Ming-Hsien Yeh ${ }^{1,2,3}$, Hanoch Livneh ${ }^{4}$, Bor-Chyuan Chen ${ }^{1}$, I-Hsin Lin², Ming-Chi Lu ${ }^{5,6}$, Tzung-Yi Tsai ${ }^{7,8,9^{*}}$ and Chia-Chou Yeh ${ }^{1,2,3^{*}}$

\begin{abstract}
Background: Patients with hypertension (HTN) reportedly have a higher risk of developing dementia. However, it remains unclear if use of Traditional Chinese Medicine (TCM), the most common form of complementary and alternative medicine, can help lower the risk of dementia for these patients. So the aim of the study was to investigate the effects of TCM on dementia risk among patients with hypertension.

Methods: This longitudinal cohort study used the Taiwanese National Health Insurance Research Database (NHIRD) to identify 143,382 newly diagnosed hypertension patients aged 20-90 years who received treatment between 1998 and 2007. Among them, 52,365 (36.52\%) had received TCM after the onset of hypertension (TCM users), and the remaining 91,017 patients (63.48\%) were designated as a control group (non-TCM users). All enrollees were followed until the end of 2012 to record the incidence of dementia. A Cox proportional hazards regression model was used to compute the hazard ratio (HR) of dementia in patients who received TCM.

Results: During the 15-year follow-up, 3933 TCM users and 10,316 non-TCM users developed dementia, representing an incidence rate of 8.41 and $11.55 \%$, respectively, per 1000 person-years. TCM users had a significantly reduced risk of dementia compared to non-TCM users (adjusted HR $=0.76 ; 95 \%$ confidence interval $[\mathrm{Cl}]=0.74-0.81$ ). The predominant effect was observed among those treated with TCM longer than 180 days (adjusted $\mathrm{HR}=0.65 ; 95 \% \mathrm{Cl}=0.62-0.69$ ). Among the commonly used TCM products, Tian-Ma-Gou-Teng-Yin, Dan-Shen (Radix Salviae Miltiorrhizae), Chuan-Niu-Xi (Radix Cyathulae), Ge-Gen (Radix Puerariae), Jia-Wei-Xiao-Yao-San, and Jue-Ming-Zi (Semen Cassiae) were significantly associated with a lower risk of dementia.
\end{abstract}

Conclusions: Results from this population-based study support the effects of TCM on reducing dementia risk, which may provide a reference for dementia prevention strategies.

Keywords: Cohort study, Dementia, Traditional Chinese medicine, Hypertension

\footnotetext{
* Correspondence: dm732024@tzuchi.com.tw; yehcc0530@gmail.com ${ }^{7}$ Department of Medical Research, Dalin Tzuchi Hospital, The Buddhist Tzuchi Medical Foundation, 2 Minsheng Road, Dalin Township, Chiayi 62247, Taiwan 'Department of Chinese Medicine, Dalin Tzuchi Hospital, The Buddhist

Tzuchi Medical Foundation, 2 Minsheng Road, Dalin Township, Chiayi 62247,

Taiwan

Full list of author information is available at the end of the article
} 


\section{Background}

Dementia is characterized by impairment of cognition involving learning, memory, language, executive function, complex attention, perceptual-motor skills, and social cognition [1]. In 2015, 46.8 million people worldwide had dementia, and 9.9 million new cases are reported every year [2]. The occurrence and irreversibility of this illness pose a heavy burden on society and families. The estimated global costs of dementia care have increased from $\$ 604$ billion in 2010 to $\$ 818$ billion in 2015 (in US dollars), representing an increase of 35.4\% [2]. Although acetylcholinesterase inhibitors and N-methyl-D-aspartate (NMDA) receptor antagonists have been effective for enabling patients to maintain global function, there is currently no treatment to halt or reverse the degenerative progression of dementia [3].

Nowadays, Traditional Chinese Medicine (TCM) has become increasingly popular as an alternative treatment for several critical illnesses, such as diabetes mellitus [4], atopic dermatitis [5], cancer [6], fractures [7], or vertigo [8]. Although previous research has indicated that several TCM herbs have shown potential benefits for dementia intervention [9], the evidence of long-term effects of TCM on dementia risk is still limited.

Hypertension (HTN), a common chronic disorder, is a critical challenge in global public health. It is noteworthy that HTN has been consistently associated with an increased risk of dementia in most cross-sectional and longitudinal cohort studies, and appears to be more relevant when it presents in middle age rather than later in life [10-12]. Results of previous studies have demonstrated that TCM can lower blood pressure and modify vascular risk factors in patients with $\operatorname{HTN}[13,14]$, which may be related to dementia $[15,16]$. However, to our knowledge, there is a paucity of information on the benefit of TCM for dementia risk, in particular for subjects with HTN. To address this concern, we studied claims data from the Taiwanese National Health Insurance Research Database (NHIRD) to determine the effect of TCM services on subsequent risk of dementia among HTN individuals. The results of this study may serve as a reference for further pharmacological studies and clinical trials.

\section{Methods}

\section{Data source}

This retrospective cohort study used Taiwan's Longitudinal Health Insurance Database (LHID), which is maintained by the National Health Insurance Administration and available to Taiwanese researchers. In 1995, Taiwan launched a single-payer national health insurance program designed to remove financial barriers to medical care for all legal residents. As of 2010, over 99\% of Taiwan's population have been enrolled in this program
[17]. The LHID, a sub-dataset of the NHI program, is made up of data from 1 million randomly sampled Taiwanese citizens alive in 2000. We collected all medical records available for these individuals from 1997 to 2012. Because a multistage stratified systematic sampling method was used, no statistically significant differences in sex or age existed between the 1 million insured individuals and the general population [17]. This database contains all NHI enrollment files, claims data, and the registry for prescription drugs, which thus provides comprehensive pharmacological utilization information for subjects covered by the insurance program. To date, more than 300 published papers have utilized this deidentified secondary data.

This study was conducted in accordance with the Helsinki Declaration, and it was also evaluated and approved by the local Institutional Review Board and Ethics Committee of Buddhist Dalin Tzu Chi Hospital, Taiwan (No. B10004021-1). Since the LHID files contain only de-identified secondary data, the review board waived the requirement for obtaining informed consent from the patients.

\section{Study population}

The participant selection method is shown in Fig. 1. All diagnoses in this insurance claims data were coded with

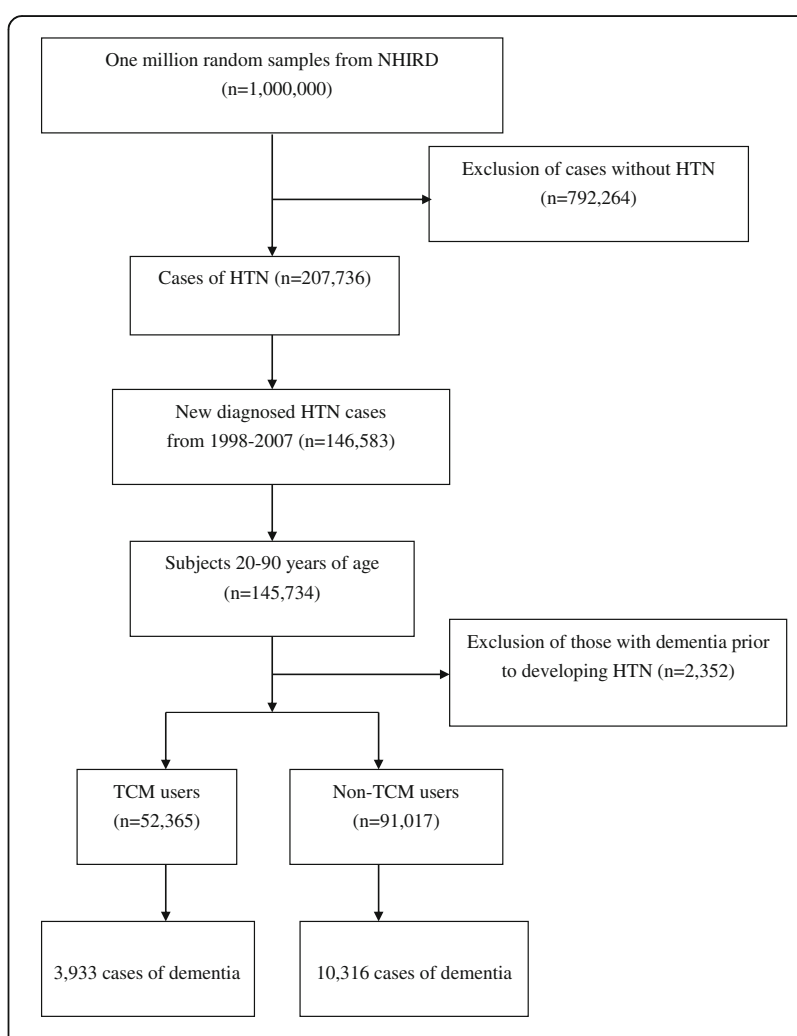

Fig. 1 A flowchart about the selection and follow-up of study subjects 
the International Classification of Disease, 9th Revision, Clinical Modification (ICD-9-CM). With it, we identified patients 20 to 90 years of age with newly diagnosed HTN within the 1998-2007 time period (ICD-9-CM codes: 401-405) as the study cohort. To reduce concern about disease misclassification, we selected only those cases where at least three diagnoses were made during outpatient visits or cases where patients were admitted to a hospital with a primary diagnosis of HTN within the observational period $(n=145,734)$. Patients with HTN who were followed for less than 3 months or who had a prior diagnosis of dementia before the first-time HTN diagnosis were excluded $(n=2352)$. Patients with dementia were identified if they had at least two treatment claims for dementia during outpatient visits or if they had been hospitalized with dementia for ICD-9-CM codes 290, 294, or 331. A final group of 143,382 subjects with HTN were included in the data analysis.

In Taiwan, only certified Chinese medicine physicians are entitled to provide TCM services. We used the frequency of visits for TCM to verify the TCM exposure of each study subject. HTN subjects who received TCM for more than 30 days were considered TCM users, whereas those treated for 30 days or less were considered nonTCM users [8]. The index date of the follow-up period for HTN subjects who were classified as non-TCM users was assigned to the date of the first HTN diagnosis, whereas the index date of follow-up period for HTN cases with TCM usage was assigned to the first date of the initiation of TCM services. The end date of the follow-up period for both groups was assigned as the date of the earliest of one of the following: a diagnosis of dementia, the date of withdrawal from the insurance program, or the date of December 31, 2012.

\section{Demographic characteristics and comorbidity}

Demographic characteristics considered in this study included age, gender, income for estimating insurance payment, the availability of TCM resources, and urbanization level of the subject's residential area. The subjects' monthly incomes were stratified into 3 levels: $\leq$ New Taiwan Dollar (NTD) 17,880, NTD 17,881-NTD 43,900 , and $\geq$ NTD 43,901. Urbanization levels were divided into 3 strata: urban (levels 1-2), suburban (levels 3-4), and rural (levels 5-7) areas. Level 1 refers to the "most urbanized" and level 7 refers to the "least urbanized" communities [18]. The comorbid medical conditions, for each individual, were evaluated by using the Charlson-Deyo comorbidity index (CCI) [19]. This index is a widely used method of categorizing comorbidities of patients based on the ICD-9 diagnosis codes found in administrative data. In this study, only diagnoses given ahead of and in concurrence with the diagnosis of HTN were regarded as underlying comorbidities.

\section{Statistical analysis}

We used the chi-square test and Student's $t$-test to examine the differences in demographic characteristics and comorbidities between subjects who received and those who did not receive TCM services. Thereafter, the Cox proportional hazards regression analysis was applied to compute the hazard ratio (HR) with $95 \%$ confidence intervals $(\mathrm{CI})$ of dementia risk in association with TCM use. To test the robustness of the relationship between TCM use and dementia risk, we divided the TCM users into two subgroups: one group used TCM for 30 to 180 days, and the other group used TCM for more than 180 days. Furthermore, a stratified analysis by age and gender using Cox proportional hazards regression was also conducted to assess the relative risk of dementia among the subjects who received and did not receive TCM services. The proportional-hazards assumption was verified using plots of $\log$ (-log (survival function) vs $\log$ (time) and Schoenfeld residuals vs time. All analyses were conducted using SAS version 9.3 (SAS Institute Inc., Cary, NC, USA), at a $P<0.05$ statistically significant level.

\section{Results}

We identified 143,382 patients with HTN during the period from 1998 to 2007. Of these, 52,365 subjects received TCM services and 91,017 subjects were classified as non-TCM users. Table 1 shows the basic characteristics of the two groups. Compared to the non-TCM users, HTN patients receiving TCM services were more likely to be female and younger, to have lower monthly incomes and tobacco use, to have higher CCI scores, and to reside in an urban area (all $P<0.01$ ).

Among the 143,382 HTN subjects, 14,249 first episodes of dementia occurred; 10,316 were reported among the non-TCM users and 3933 among the TCM users during the follow-up of 892,960.27 and 467,500.55 person-years (PYs), respectively. The incidence rate of dementia was lower among TCM users than among non-TCM users (8.41 vs 11.55 , respectively, per 1000 PYs), with an adjusted HR of $0.76(95 \% \mathrm{CI}=0.74-0.81)$ (Table 2). Of note, using TCM services for more than 180 days was associated with a $35 \%$ decreased risk of dementia among HTN patients $(95 \% \mathrm{CI}=0.62-0.69)$.

As for the sex-specific risk of dementia, we discovered that both female and male HTN patients receiving TCM services had a significantly decreased risk of dementia, with an adjusted HR of $0.71(95 \% \mathrm{CI}=0.68-0.74)$ and $0.84(95 \%$ CI $=0.81-0.91)$, respectively (Table 3$)$. Additionally, because a significant interaction of age and sex in relation to TCM use occurred, we further performed a stratified analysis by age and sex to determine the effect of TCM on dementia risk. In general, use of TCM was associated with a lower risk of dementia, irrespective 
Table 1 Demographic data and selected comorbidities of the study subjects

\begin{tabular}{llll}
\hline Variables & Non-TCM users & TCM users & $P$-value \\
& $n=91,017(\%)$ & $n=52,365(\%)$ & \\
\hline Age (yr) & & & $<0.001$ \\
$\leq 50$ & $22,971(25.2)$ & $16,461(31.4)$ & \\
$>50$ & $68,046(74.8)$ & $35,904(68.6)$ & \\
Mean (SD) & $59.74(13.6)$ & $57.19(12.4)$ & $<0.001$ \\
Gender & & & $<0.001$ \\
Female & $40,794(44.8)$ & $29,597(56.5)$ & \\
Male & $50,223(55.2)$ & $22,768(43.5)$ & \\
Monthly income & & & $<0.001$ \\
Low & $39,976(43.9)$ & $21,506(41.1)$ & \\
Median & $46,352(50.9)$ & $28,123(53.7)$ & \\
High & $4689(5.2)$ & $2736(5.2)$ & \\
Residential area & & & $<0.001$ \\
$\quad$ Urban & $49,720(54.6)$ & $29,629(56.6)$ & \\
Suburban & $14,209(15.6)$ & $8426(16.1)$ & \\
Rural & $27,088(29.8)$ & $14,310(27.3)$ & \\
Tobacco use & & & \\
Yes & $352(0.4)$ & $150(0.3)$ & \\
No & $90,665(99.6)$ & $52,215(99.7)$ & \\
CCl & & & \\
Mean (SD) & $3.50(4.42)$ & & \\
\hline
\end{tabular}

TCM Traditional Chinese Medicine, SD, standard deviation; CCI Charlson-Deyo comorbidity index

of sex. The multivariable stratified analysis verified significant associations of dementia risk with TCM use among male subjects with HTN aged $\leq 50$ years (adjusted $\mathrm{HR}=0.72 ; 95 \% \mathrm{CI}=0.57-0.89$ ); for female subjects, the more beneficial effect of TCM on the risk of dementia was noted for those older than 50 years (adjusted $H R=0.70$; 95\% CI $=0.67-0.75$ ) (Table 3).

The most commonly prescribed TCMs for patients with HTN are summarized in Table 4. Of the 15 most common TCMs, 6 were herbal formulas and 9 were single herbs. Tian-Ma-Gou-Teng-Yin (TMGTY) was the most used herbal formula, followed by Dan-Shen and
Gou-Teng-San. Of these TCMs, Tian-Ma-Gou-Teng-Yin, Dan-Shen, Chuan-Niu-Xi, Ge-Gen, Jia-Wei-Xiao-YaoSan, and Jue-Ming-Zi were found to be significantly related to a lower risk of dementia (Table 5).

\section{Discussion}

To our knowledge, this is the first population-based cohort study to address the influence of TCM on the risk of dementia among patients with HTN. The study results provide more robust findings about the effects of TCM in this patient population, and may allow clinicians to choose the most appropriate treatment for HTN individuals.

Results of this 15-year follow-up study showed that subjects with HTN who received TCM services exhibited a $24 \%$ reduced risk of dementia as compared to those who did not receive TCM services. In addition, the use of TCM services for more than 180 days was associated with a 35\% decreased risk of developing dementia. Furthermore, the stratified analysis supported that the TCM intervention significantly reduced the risk of dementia in females more than for males, with adjusted HR of 0.71 and 0.84 , respectively. One contributing factor might be that Taiwanese females often exhibit better adherence behaviors than males [20], thus preventing their disease from worsening.

The findings of this study also showed that TCM services may result in fewer effects on females younger than 50 years of age compared to males of similar age. We speculate that younger women may benefit from higher estrogen levels, which could decrease serum low-density lipoprotein-cholesterol (LDL-C) levels and curb coronary thrombosis and atherosclerosis by regulating vascular smooth muscle and endothelial cells [21]. This biochemical benefit may lessen the corresponding effect of TCM services. Taken together, these results clearly demonstrate that earlier and longer use of TCM among HTN patients is associated with reduction of dementia risk.

The two principal types of dementia, Alzheimer's disease $(\mathrm{AD})$ and vascular dementia $(\mathrm{VaD})$, account for about 70 and $15 \%$ of cases of dementia, respectively [22]. Reducing the amount of beta-amyloid protein $(\mathrm{A} \beta)$ and tau protein in the brain and modulating $A \beta$ toxicity, including inflammatory response, oxidative stress, and

Table 2 Risk of dementia for HTN subjects with and without TCM

\begin{tabular}{llllll}
\hline Patient group & Event & PYs & Incidence & Crude HR (95\% Cl) & Adjusted HR (95\% Cl) \\
\hline Non-TCM users & 10,316 & $892,960.27$ & 11.55 & 1 & 1 \\
TCM users & 3933 & $467,500.55$ & 8.41 & $0.73(0.71-0.76)$ & $0.76(0.74-0.81)$ \\
$\begin{array}{l}\text { TCM use within } \\
\text { 30-180 days }\end{array}$ & 2706 & $289,829.43$ & 9.34 & $0.82(0.78-0.85)$ & $0.86(0.82-0.90)$ \\
TCM use for more than 180 days & 1277 & $177,671.13$ & 6.91 & $0.60(0.57-0.64)$ & $0.65(0.62-0.69)$ \\
\hline
\end{tabular}

TCM Traditional Chinese Medicine, $P Y s$ per 1000 person-years, $H R$ hazard ratio, $C l$ confidence interval

${ }^{a}$ Model adjusted for age, gender, urbanization level, monthly income, and $\mathrm{CCl}$ scores 
Table 3 Incidence and dementia risk for HTN patients with and without TCM in the stratification of sex and age

\begin{tabular}{|c|c|c|c|c|c|c|c|c|}
\hline \multirow[t]{2}{*}{ Variables } & \multicolumn{3}{|c|}{ Non-TCM users } & \multicolumn{3}{|c|}{ TCM users } & \multirow{2}{*}{$\begin{array}{l}\text { Crude HR } \\
(95 \% \mathrm{Cl})\end{array}$} & \multirow{2}{*}{$\begin{array}{l}\text { Adjusted HR } \\
(95 \% \mathrm{Cl})\end{array}$} \\
\hline & Case & PYS & Incidence & Case & PYS & Incidence & & \\
\hline \multicolumn{9}{|l|}{ Female } \\
\hline$\leq 50 \mathrm{yr}$ & 148 & $81,138.18$ & 1.82 & 111 & $79,715.46$ & 1.39 & $\begin{array}{l}0.77 \\
(0.60-0.98)\end{array}$ & $\begin{array}{l}0.76^{\mathrm{a}} \\
(0.59-0.97)\end{array}$ \\
\hline$>50 \mathrm{yr}$ & 4949 & $320,644.53$ & 15.43 & 2104 & $188,214.45$ & 11.18 & $\begin{array}{l}0.73 \\
(0.70-0.77)\end{array}$ & $\begin{array}{l}0.70^{\mathrm{a}} \\
(0.67-0.75)\end{array}$ \\
\hline All & 5097 & $401,782.71$ & 12.69 & 2215 & $267,926.61$ & 8.27 & $\begin{array}{l}0.66 \\
(0.63-0.69)\end{array}$ & $\begin{array}{l}0.71^{\mathrm{b}} \\
(0.68-0.74)\end{array}$ \\
\hline \multicolumn{9}{|l|}{ Male } \\
\hline$\leq 50 \mathrm{yr}$ & 307 & $139,732.62$ & 2.20 & 107 & $65,359.01$ & 1.64 & $\begin{array}{l}0.75 \\
(0.59-0.93)\end{array}$ & $\begin{array}{l}0.72^{\mathrm{a}} \\
(0.57-0.89)\end{array}$ \\
\hline$>50 \mathrm{yr}$ & 4912 & $351,444.93$ & 13.98 & 1611 & $134,211.63$ & 12.00 & $\begin{array}{l}0.85 \\
(0.81-0.91)\end{array}$ & $\begin{array}{l}0.87^{\mathrm{a}} \\
(0.83-0.92)\end{array}$ \\
\hline All & 5219 & $491,177.56$ & 10.63 & 1718 & $199,570.64$ & 8.61 & $\begin{array}{l}0.81 \\
(0.77-0.86)\end{array}$ & $\begin{array}{l}0.84^{b} \\
(0.81-0.91)\end{array}$ \\
\hline
\end{tabular}

TCM Traditional Chinese Medicine, $P Y s$ person-years, $H R$ hazard ratio, $C l$ confidence interval

${ }^{a}$ Model adjusted for urbanization level, monthly income, and $\mathrm{CCl}$ scores

${ }^{\mathrm{b}}$ Model adjusted for age, urbanization level, monthly income, and $\mathrm{CCl}$ scores

neuronal apoptosis, have been considered the most promising therapeutic strategies available for controlling the progression of dementia [23, 24].

In our study, six TCMs were found to be significantly related to a lower risk of dementia, and these have been reported to exert most of the neuroprotective activities mentioned above. Additionally, most of these TCMs have shown medicinal benefits in reducing vascular risk factors of $\mathrm{AD}$ and $\mathrm{VaD}$, and thus they may also help reduce the risk of dementia $[15,16]$. The possible pharmacological mechanisms of the most common TCMs for the treatment of patients with HTN are summarized in Tables 4 and 5 .

We discovered that the most commonly prescribed herbal formula, Tian-Ma-Gou-Teng-Yin (TMGTY), was used in TCM for relieving symptoms related to high blood pressure, such as headaches and dizziness. Results of previous studies have shown that TMGTY has significant effects on a variety of HTN-caused cardiovascular diseases [14]. Recent scientific evidence has also demonstrated that TMGTY provides neuroprotective effects for patients with Parkinson's disease [25]. TMGTY has also been found to inhibit the NMDA receptor. This leads to a reduction in necrosis and apoptosis in neuronal cells through a variety of pharmacological effects, including anti-inflammatory, antioxidative, and antiapoptotic activities [26]. Moreover, the components of TMGTY could decrease the activity of acetylcholinesterase and show potent anti-aggregation effects on $A \beta$ proteins [27, 28].

Dan-Shen (Radix Salviae Miltiorrhizae) and Ge-Gen (Radix Puerariae) are two herbs frequently used for the treatment of angina and other cardiac symptoms in
TCM. Recent studies have demonstrated their cardioprotective and anti-atherosclerosis effects. Results of pharmacological studies suggest that these herbs can control high blood pressure, lower serum lipids, and improve microcirculation [29]. Dan-Shen demonstrated neuroprotective effects [29], including inhibition of $A \beta$ aggregation, oligomerization, and fibril formation through upregulation of activity of alpha-secretases [30]. Dan-Shen also acts as an acetylcholinesterase inhibitor [31], and it could improve age-related oxidative stress and inflammatory response [32], protect endothelial cells from hydrogen peroxide damage, and inhibit apoptosis [33]. Ge-Gen was found to show potential medicinal benefits in diabetes and cardiovascular and cerebrovascular diseases [34], and it could alleviate neurological deficits and improve learning and memory after ischemia/reperfusion-induced cerebral microcirculatory disturbances [35]. Recent studies have also demonstrated that Ge-Gen could inhibit $A \beta$ plaque accumulation [36], suppress tau protein expression [37], and antagonize neuronal apoptosis induced by oxidative stress [38].

Chuan-Niu-Xi (Radix Cyathulae) has demonstrated various beneficial pharmacological activities, including analgesic, immunostimulant, antitumor, anti-inflammatory, and antiaging effects. Its use removes blood stasis, restores menstrual flow, and induces diuresis for treating stranguria. It also has antioxidant qualities [39].

Jia-Wei-Xiao-Yao-San is widely used to relieve emotional and neuropsychological disorders such as depression, stress [40], and dyskinesia in patients with schizophrenia [41]. Researchers have also shown that Jia-Wei-Xiao-Yao-San can reduce tremors of antipsychotic-induced parkinsonism [42], relieve migraine headache [43], and improve the 
Chen et al. BMC Complementary and Alternative Medicine (2017) 17:178

Page 6 of 10

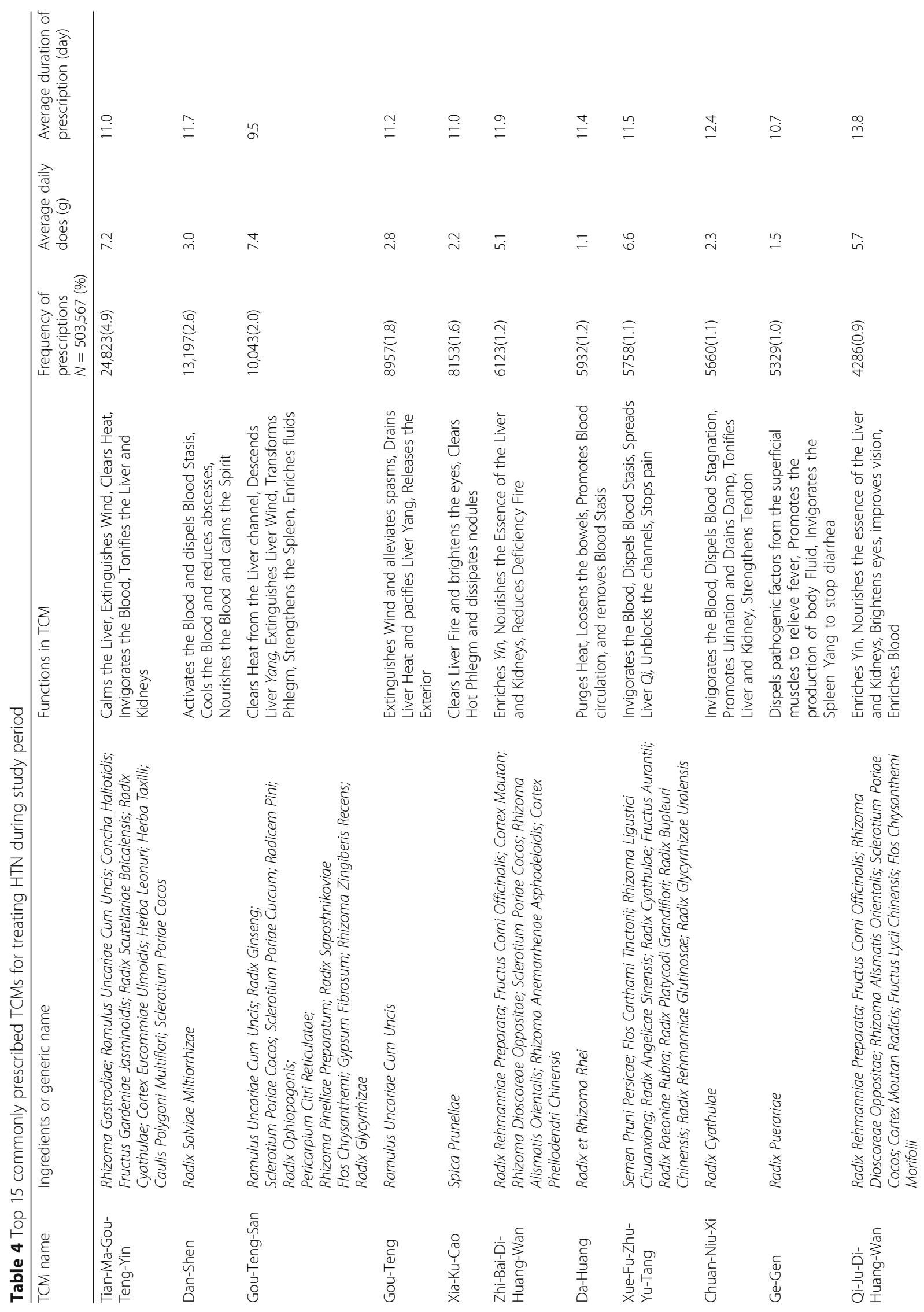




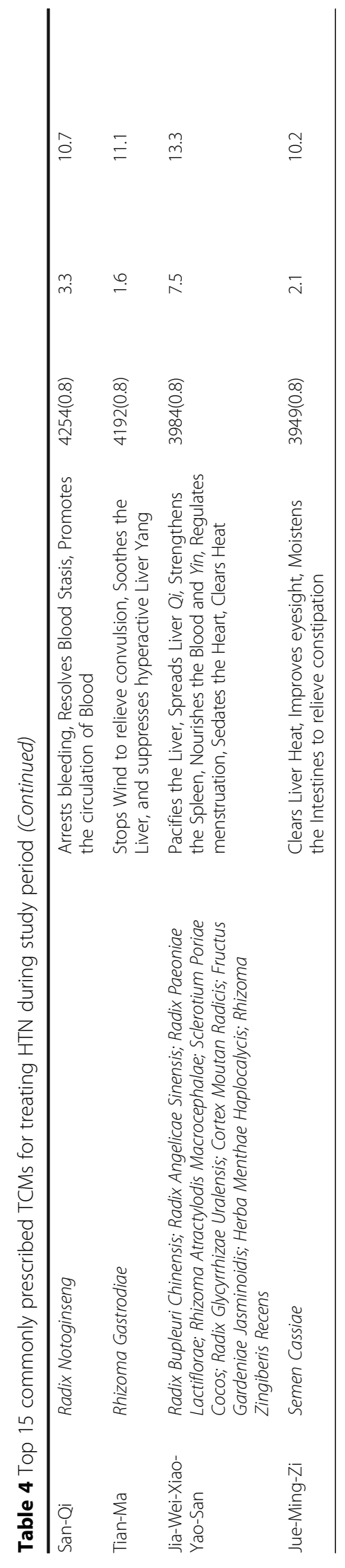


Table 5 Risk of dementia in relation to the top 15 commonly used TCMs

\begin{tabular}{lll}
\hline TCM name & $\begin{array}{l}\text { Crude HR } \\
(95 \% \mathrm{Cl})\end{array}$ & $\begin{array}{l}\text { Adjusted HR } \\
(95 \% \mathrm{Cl})\end{array}$ \\
\hline Tian-Ma-Gou-Teng-Yin ${ }^{\text {a,c,d,e,f,g,h,i }}$ & $0.77(0.68-0.86)$ & $0.82(0.76-0.95)^{k}$ \\
Dan-Shen a,d,e,f,g,h,i & $0.71(0.61-0.83)$ & $0.80(0.69-0.92)^{k}$ \\
Gou-Teng-San & $0.78(0.65-0.92)$ & $0.90(0.74-1.07)$ \\
Gou-Teng & $0.75(0.61-0.90)$ & $0.89(0.75-1.08)$ \\
Xia-Ku-Cao & $0.70(0.56-0.86)$ & $0.79(0.60-1.02)$ \\
Zhi-Bai-Di-Huang-Wan & $0.88(0.68-1.10)$ & $0.97(0.76-1.23)$ \\
Da-Huang & $0.89(0.67-1.15)$ & $0.92(0.70-1.20)$ \\
Xue-Fu-Zhu-Yu-Tang & $0.82(0.67-0.99)$ & $0.91(0.75-1.21)$ \\
Chuan-Niu-Xi e,f,i & $0.73(0.58-0.92)$ & $0.79(0.62-0.98)^{k}$ \\
Ge-Gen ${ }^{k}, b, f, f, h,, i$ & $0.62(0.43-0.79)$ & $0.65(0.52-0.83)^{k}$ \\
Qi-Ju-Di-Huang-Wan & $0.87(0.68-1.12)$ & $0.85(0.66-1.09)$ \\
San-Qi & $0.85(0.66-1.10)$ & $0.89(0.69-1.19)$ \\
Tian-Ma & $0.98(0.79-1.22)$ & $0.96(0.77-1.19)$ \\
Jia-Wei-Xiao-Yao-San ${ }^{\text {a,b,i }}$ & $0.70(0.54-0.91)$ & $0.79(0.64-0.98)^{k}$ \\
Jue-Ming-Zi a,e,h,i & $0.57(0.41-0.80)$ & $0.68(0.49-0.96)^{k}$ \\
\hline
\end{tabular}

$H R$ hazard ratio, $\mathrm{Cl}$ confidence interval

Potential therapeutic effects of the most promising TCMs:

${ }^{a}$ Reduces $\beta$-amyloid protein or its toxicity

${ }^{b}$ Reduces Tau protein or its abnormal phosphorylation

Inhibits NMDA receptor

dinhibits acetylcholinesterase

${ }^{\mathrm{e}}$ Anti-inflammatory

${ }^{f}$ Antioxidant activity

${ }^{9}$ Anti-apoptosis

${ }^{\mathrm{h}}$ Neuroprotective effects

iMedicinal benefits in vascular risk factors

'Model adjusted for age, gender, urbanization level, monthly income, and $\mathrm{CCl}$ scores

${ }^{\mathrm{k}}$ Promising TCMs that were significantly related to the lower risk of dementia

survival rate of type 2 diabetes patients with HTN [44]. Moreover, the components of Jia-Wei-Xiao-Yao-San can inhibit abnormal tau phosphorylation, suppress the release of $\mathrm{A} \beta$ peptides, and decrease beta-amyloid-induced neurotoxicity $[45,46]$.

Another TCM herb, Jue-Ming-Zi (Semen Cassiae) has shown neuroprotective effects in animal models of ischemic stroke and Parkinson's disease [47]. Results of a recent study also demonstrated that this herb can ameliorate amyloid- $\beta$-induced synaptic dysfunction through antiinflammatory and Akt/GSK-3 $\beta$ pathways [48]. In our study, all of the previously mentioned TCM agents relieved the symptoms of HTN and decreased the risk of developing dementia.

Although our study is the first to recently investigate the effect of use of TCM on the risk of dementia among subjects with HTN, there are some important limitations to consider. First, identification of use of TCM and outcomes were based on three categories in the ICD-9-CM, and inaccurate diagnosis may have occurred. To minimize this error, we only selected subjects with either HTN or dementia after they were recorded as having at least three outpatient visits reporting consistent diagnoses or one inpatient admission for HTN. It should also be noted that the NHI of Taiwan randomly samples claims from hospitals, interviews patients, and reviews medical charts to verify the accuracy of medical records. Second, we could not account for other confounding factors, such as the use of tobacco and alcohol, physical activity, dietary preferences, social network relationships, coping strategies, or educational level, which were unavailable from the claims data. Further studies controlling for those untested factors are recommended to assess whether the present findings are replicable among other demographically and geographically diverse groups. Third, we were unable to contact the enrolled patients directly about the use of Chinese herbs due to the anonymity of identification numbers in the database. Even so, we were still able to demonstrate the benefit from TCM use. Nearly $95 \%$ of dosing frequencies in Chinese herbs are typically only used for one week in clinical practice, so those who continued to receive the same prescriptions for a longer period were therefore likely to have used the prescribed medication [49]. Fourth, the NHI program only pays for TCMs prescribed by Chinese medicine physicians, not over-the-counter TCMs. Therefore, the use of TCM may be underestimated. However, the NHI covers TCM prescriptions (the concentrated herbal powder) manufactured by GMP-certified pharmaceutical companies in Taiwan. Within the NHI program, the copayment for visiting a TCM clinic is only approximately 10 USD, which may greatly enhance the accessibility to TCM services. Fifth, findings from any retrospective cohort study are generally less sound than these from randomized trials because cohort study designs are subject to various biases related to uncontrolled confounding effects. Despite our careful attention to the study design, employing adequate control of confounding factors, unpredictable biases could still remain if they stem from unmeasured or unknown confounders. Notwithstanding these limitations, the strengths of this study must also be acknowledged and these included the immediate availability of data, the comprehensiveness of the database, and the statistical power derived from the samples' large sizes. In addition, this retrospective 15-year cohort study allowed us to examine in detail the association of TCM usage with the subsequent risk of dementia, and the corresponding findings could serve as a reference for future treatments.

\section{Conclusions}

The results of this population-based, retrospective cohort study show that the use of TCM during treatment of HTN was associated with a $24 \%$ lower risk of developing dementia compared to the risk among non-TCM users. TCM users had a significantly reduced risk of 
dementia compared to non-TCM users (adjusted $\mathrm{HR}=0.76 ; 95 \% \mathrm{CI}=0.74-0.81$ ). The predominant effect was observed among those treated with TCM longer than 180 days (adjusted $\mathrm{HR}=0.65 ; 95 \% \mathrm{CI}=0.62-0.69$ ). These findings could serve as a reference for healthcare providers, in helping to establish more effective therapeutic interventions to improve the prognosis of patients with HTN and prevent subsequent dementia.

\section{Abbreviations}

Aß: beta-amyloidADAlzheimer's diseaseCCICharlson-Deyo comorbidity indexCIConfidence intervalGMPGood Manufacturing PracticeHRHazard ratioHTNHypertensionLHIDLongitudinal Health Insurance DatabaseNHINational Health InsuranceNHIRDNational Health Insurance Research DatabaseNMDANmethyl-D-aspartateNTDNew Taiwan DollarPYsPer 1000 person-yearsSDStandard deviationTCMTraditional Chinese MedicineTMGTYTian-Ma-Gou-TengYinUSDUnited States DollarVaDVascular dementia

\section{Acknowledgements}

The study is based in part on data from the National Health Insurance Research Database provided by the Bureau of National Health Insurance, Department of Health and managed by the National Health Research Institutes, in Taiwan. The interpretation and conclusions contained herein do not represent those of the Bureau of National Health Insurance, Department of Health or National Health Research Institutes. This research was supported by Dalin Tzuchi Hospital (Grants DTCRD103 (2)-E-05). KH Chen and MH Yeh contributed equally to this work. Both TY Tsai and CC Yeh were corresponding authors.

\section{Funding}

This study was supported by a research grant provided by Dalin Tzuchi Hospital (Grant No: DTCRD 103(2)-E-05). The funding agent plays no role in study design data collection, and data analyses.

\section{Authors' contributions}

$\mathrm{KH}$ Chen and $\mathrm{MH}$ Yeh were involved in the study design and drafted the manuscript. MC Lu contributed to data analysis and revised the manuscript. $\mathrm{H}$ Livneh and BC Chen contributed to the interpretation of data and provided comments on the final draft of the manuscript. IH Lin and MC Lu provided administrative support and comments on the manuscript drafts. TY Tsai and CC Yeh were responsible for the study conception, design, data analysis, and drafting of the manuscript. All authors read and approved the final manuscript.

\section{Availability of data and materials}

The dataset is available from Taiwan's National Health Research Institutes (http://nhird.nhri.org.tw)

\section{Competing interests}

The authors declare that they have no competing interests.

\section{Consent for publication}

All authors have read and agreed to all the contents for publication.

\section{Ethics approval and consent to participate}

This study was conducted in accordance with the Helsinki Declaration, and it was also evaluated and approved by the local Institutional Review Board and Ethics Committee of Buddhist Dalin Tzu Chi Hospital, Taiwan (No. B10004021-1).

\section{Author details}

'Department of Chinese Medicine, Dalin Tzuchi Hospital, The Buddhist Tzuchi Medical Foundation, 2 Minsheng Road, Dalin Township, Chiayi 62247, Taiwan. ${ }^{2}$ School of Post-Baccalaureate Chinese Medicine, Tzu Chi University, 701 Jhongyang Road Section 3, Hualien 97004, Taiwan. ${ }^{3}$ School of Chinese Medicine, China Medical University, Taichung 40402, Taiwan. ${ }^{4}$ Rehabilitation Counseling Program, Portland State University, Portland, OR 97207-0751, USA. ${ }^{5}$ Division of Allergy, Immunology and Rheumatology, Dalin Tzuchi Hospital, The Buddhist Tzuchi Medical Foundation, 2 Minsheng Road, Dalin Township, Chiayi 62247, Taiwan. ${ }^{6}$ School of Medicine, Tzu Chi University, 701 Jhongyang Road Section 3, Hualien 97004, Taiwan. ${ }^{7}$ Department of Medical Research, Dalin Tzuchi Hospital, The Buddhist Tzuchi Medical Foundation, 2
Minsheng Road, Dalin Township, Chiayi 62247, Taiwan. ${ }^{8}$ Department of Environmental and Occupational Health, College of Medicine, National Cheng Kung University, 138 Sheng-Li Road, Tainan 70428, Taiwan.

${ }^{9}$ Department of Nursing, Tzu Chi University of Science and Technology, 880 Chien-Kuo Road Section 2, Hualien 97004, Taiwan.

Received: 10 October 2016 Accepted: 11 March 2017

Published online: 29 March 2017

\section{References}

1. American Psychiatric Association. Diagnostic and Statistical Manual of Mental Disorders. 5th ed. Washington, DC: Author; 2013.

2. Alzheimer's Disease International. World Alzheimer Report. In.: 2015.

3. Raina $P$, Santaguida $P$, Ismaila A, Patterson C, Cowan D, Levine $M$, et al. Effectiveness of cholinesterase inhibitors and memantine for treating dementia: evidence review for a clinical practice guideline. Ann Intern Med. 2008;148:379-7.

4. Hsu PC, Tsai YT, Lai JN, Wu CT, Lin SK, Huang CY. Integrating traditional Chinese medicine healthcare into diabetes care by reducing the risk of developing kidney failure among type 2 diabetic patients: a populationbased case control study. J Ethnopharmacol. 2014;156:358-64.

5. Chen YC, Lin YH, Hu S, Chen HY. Characteristics of traditional Chinese medicine users and prescription analysis for pediatric atopic dermatitis: a population-based study. BMC Complement Altern Med. 2016:16:173.

6. Fleischer T, Chang TT, Chiang JH, Hsieh CY, Sun MF, Yen HR. Integration of Chinese herbal medicine therapy improves survival of patients with chronic lymphocytic leukemia: a nationwide population-based cohort study. Medicine. 2016;95:e3788

7. Liao HH, Yeh CC, Lin CC, Chen BC, Yeh MH, Chang KM, et al. Prescription patterns of Chinese herbal products for patients with fractures in Taiwan: a nationwide population-based study. J Ethnopharmacol. 2015;173:11-9.

8. Tsai TY, Li CY, Livneh H, Lin IH, Lu MC, Yeh CC. Decreased risk of stroke in patients receiving traditional Chinese medicine for vertigo: a populationbased cohort study. J Ethnopharmacol. 2016;184:138-43.

9. Lin Z, Gu J, Xiu J, Mi T, Dong J, Tiwari JK. Traditional chinese medicine for senile dementia. Evid Based Complement Alternat Med. 2012;2012:692621.

10. Gottesman RF, Schneider AL, Albert M, Alonso A, Bandeen-Roche K, Coker L, et al. Midlife hypertension and 20-year cognitive change: the atherosclerosis risk in communities neurocognitive study. JAMA Neurol. 2014;71:1218-27.

11. Norton S, Matthews FE, Barnes DE, Yaffe K, Brayne C. Potential for primary prevention of Alzheimer's disease: an analysis of population-based data. Lancet Neurol. 2014;13:788-94.

12. Freitag MH, Peila R, Masaki K, Petrovitch $H$, Ross GW, White LR, et al. Midlife pulse pressure and incidence of dementia: the honolulu-Asia aging study. Stroke 2006;37:33-7.

13. Xiong $X$, Wang $P$, Zhang $Y$, Li X. Effects of traditional Chinese patent medicine on essential hypertension: a systematic review. Medicine. 2015;94:e442.

14. Zhang G, Yang G, Deng $Y$, Zhao X, Yang $Y$, Rao J, et al. Ameliorative effects of Xue-Fu-Zhu-Yu decoction. Tian-Ma-Gou-Teng-Yin and Wen-Dan decoction on myocardial fibrosis in a hypertensive rat mode BMC Complement Altern Med. 2016;16:56.

15. Alonso A, Mosley Jr TH, Gottesman RF, Catellier D, Sharrett AR, Coresh J. Risk of dementia hospitalisation associated with cardiovascular risk factors in midlife and older age: the Atherosclerosis Risk in Communities (ARIC) study. J Neurol Neurosurg Psychiatry. 2009:80:1194-201.

16. Debette S, Seshadri S, Beiser A, Au R, Himali JJ, Palumbo C, et al. Midlife vascular risk factor exposure accelerates structural brain aging and cognitive decline. Neurology. 2011;77:461-8.

17. National Health Research Institute. Longitudinal Health Insurance Research Database. 2011.

18. Liu CY, Hung YT, Chuang YL, Chen YJ, Weng WS, Liu JS, et al. Incorporating development stratification of Taiwan townships into sampling design of large scale health interview survey. J Health Manag. 2006;4:1-22.

19. Deyo RA, Cherkin DC, Ciol MA. Adapting a clinical comorbidity index for use with ICD-9-CM administrative databases. J Clin Epidemiol. 1992;45:613-9.

20. Shih CC, Liao CC, Su YC, Tsai CC, Lin JG. Gender differences in traditional Chinese medicine use among adults in Taiwan. PLoS One. 2012;7:e32540.

21. Moroney JT, Tang MX, Berglund L, Small S, Merchant C, Bell K, et al. Lowdensity lipoprotein cholesterol and the risk of dementia with stroke. JAMA. 1999:282:254-60.

22. Ritchie K, Lovestone S. The dementias. Lancet. 2002;360:1759-66. 
23. Lloret A, Badia MC, Giraldo E, Ermak G, Alonso MD, Pallardo FV, et al. Amyloid-beta toxicity and tau hyperphosphorylation are linked via RCAN1 in Alzheimer's disease. J Alzheimers Dis. 2011;27:701-9.

24. Xian YF, Mao QQ, Wu JC, Su ZR, Chen JN, Lai XP, et al. Isorhynchophylline treatment improves the amyloid-beta-induced cognitive impairment in rats via inhibition of neuronal apoptosis and tau protein hyperphosphorylation. J Alzheimers Dis. 2014;39:331-46.

25. Liu LF, Song JX, Lu JH, Huang YY, Zeng Y, Chen LL, et al. Tianma Gouteng Yin, a traditional Chinese medicine decoction, exerts neuroprotective effects in animal and cellular models of Parkinson's disease. Sci Rep. 2015;5:16862.

26. Chik SC, Or TC, Luo D, Yang CL, Lau AS. Pharmacological effects of active compounds on neurodegenerative disease with gastrodia and uncaria decoction, a commonly used poststroke decoction. ScientificWorldJournal. 2013;2013:896873.

27. Huang GB, Zhao T, Muna SS, Jin HM, Park JI, Jo KS, et al. Therapeutic potential of Gastrodia elata Blume for the treatment of Alzheimer's disease. Neural Regen Res. 2013;8:1061-70.

28. Fujiwara H, Iwasaki K, Furukawa K, Seki T, He M, Maruyama M, et al. Uncaria rhynchophylla, a Chinese medicinal herb, has potent antiaggregation effects on Alzheimer's beta-amyloid proteins. J Neurosci Res. 2006;84:427-33.

29. Tam WY, Chook P, Qiao M, Chan LT, Chan TY, Poon YK, et al. The efficacy and tolerability of adjunctive alternative herbal medicine (Salvia miltiorrhiza and Pueraria lobata) on vascular function and structure in coronary patients. J Altern Complement Med. 2009;15:415-21.

30. Mei Z, Zhang F, Tao L, Zheng W, Cao Y, Wang Z, et al. Cryptotanshinone, a compound from Salvia miltiorrhiza modulates amyloid precursor protein metabolism and attenuates beta-amyloid deposition through upregulating alpha-secretase in vivo and in vitro. Neurosci Lett. 2009;452:90-5.

31. Zhou Y, Li W, Xu L, Chen L. In Salvia miltiorrhiza, phenolic acids possess protective properties against amyloid beta-induced cytotoxicity, and tanshinones act as acetylcholinesterase inhibitors. Environ Toxicol Pharmacol. 2011;31:443-52.

32. Park CH, Kim DH, Park MH, Kim MK, Kim ND, Kim CM, et al. Chinese prescription kangen-karyu and salviae miltiorrhizae radix improve age-related oxidative stress and inflammatory response through the PI3K Akt or MAPK pathways. Am J Chin Med. 2014:42:987-1005

33. Yang GD, Zhang $H$, Lin $R$, Wang WR, Shi XL, Liu Y, et al. Down-regulation of CD40 gene expression and inhibition of apoptosis with Danshensu in endothelial cells. Basic Clin Pharmacol Toxicol. 2009;104:87-92.

34. Wong KH, Li GQ, Li KM, Razmovski-Naumovski V, Chan K. Kudzu root: traditional uses and potential medicinal benefits in diabetes and cardiovascular diseases. J Ethnopharmacol. 2011;134:584-607.

35. Sun K, Fan J, Han J. Ameliorating effects of traditional Chinese medicine preparation, Chinese materia medica and active compounds on ischemia/ reperfusion-induced cerebral microcirculatory disturbances and neuron damage. Acta Pharm Sin B. 2015;5:8-24.

36. Dong XH, Gao WJ, Kong WN, Xie HL, Peng Y, Shao TM, et al. Neuroprotective effect of the active components of three Chinese herbs on brain iron load in a mouse model of Alzheimer's disease. Exp Ther Med. 2015:9:1319-27.

37. Anukulthanakorn K, Parhar IS, Jaroenporn S, Kitahashi T, Watanbe G, Malaivijitnond S. Neurotherapeutic effects of pueraria mirifica extract in early- and late-stage cognitive impaired rats. Phytother Res. 2016;30:929-39.

38. Zhang H, Liu Y, Lao M, Ma Z, Yi X. Puerarin protects Alzheimer's disease neuronal cybrids from oxidant-stress induced apoptosis by inhibiting prodeath signaling pathways. Exp Gerontol. 2011:46:30-7.

39. Han X, Shen S, Liu T, Du X, Cao X, Feng H, et al. Characterization and antioxidant activities of the polysaccharides from radix cyathulae officinalis kuan. Int J Biol Macromol. 2015;72:544-52.

40. Tanaka M, Satou T, Koike K. Anxiolytic-like effect of Shigyakusan extract with low side effects in mice. J Nat Med. 2013;67:862-6.

41. Lee JG, Shin BS, Lee YC, Park SW, Kim YH. Clinical effectiveness of the Kampo medicine kamishoyosan for adjunctive treatment of tardive dyskinesia in patients with schizophrenia: a 16-week open trial. Psychiatry Clin Neurosci. 2007:61:509-14.

42. Ishikawa T, Funahashi T, Kudo J. Effectiveness of the Kampo kami-shoyo-san (TJ-24) for tremor of antipsychotic-induced parkinsonism. Psychiatry Clin Neurosci. 2000;54:579-82.

43. Chang YY, Tsai YT, Lai JN, Yeh CH, Lin SK. The traditional Chinese medicine prescription patterns for migraine patients in Taiwan: a population-based study. J Ethnopharmacol. 2014;151:1209-17.
44. Lin YJ, Ho TJ, Yeh YC, Cheng CF, Shiao YT, Wang CB, et al. Chinese herbal medicine treatment improves the overall survival rate of individuals with hypertension among type 2 diabetes patients and modulates in vitro smooth muscle cell contractility. PLoS One. 2015;10:e0145109.

45. Lee TH, Park SH, You MH, Lim JH, Min SH, Kim BM: A potential therapeutic effect of Saikosaponin C as a novel dual-target anti-Alzheimer agent. J Neurochem 2015. [Epub ahead of print].

46. Zhang Z, Zhao R, Qi J, Wen S, Tang Y, Wang D. Inhibition of glycogen synthase kinase-3beta by angelica sinensis extract decreases beta-amyloidinduced neurotoxicity and tau phosphorylation in cultured cortical neurons. J Neurosci Res. 2011;89:437-47.

47. Ju MS, Kim HG, Choi JG, Ryu JH, Hur J, Kim YJ, et al. Cassiae semen, a seed of Cassia obtusifolia, has neuroprotective effects in Parkinson's disease models. Food Chem Toxicol. 2010;48:2037-44.

48. Yi JH, Park HJ, Lee S, Jung JW, Kim BC, Lee YC, et al. Cassia obtusifolia seed ameliorates amyloid beta-induced synaptic dysfunction through antiinflammatory and Akt/GSK-3beta pathways. J Ethnopharmacol. 2016;178:50-7.

49. Lai MN, Wang SM, Chen PC, Chen YY, Wang JD. Population-based casecontrol study of Chinese herbal products containing aristolochic acid and urinary tract cancer risk. J Natl Cancer Inst. 2010;102:179-86.

\section{Submit your next manuscript to BioMed Central and we will help you at every step:}

- We accept pre-submission inquiries

- Our selector tool helps you to find the most relevant journal

- We provide round the clock customer support

- Convenient online submission

- Thorough peer review

- Inclusion in PubMed and all major indexing services

- Maximum visibility for your research

Submit your manuscript at www.biomedcentral.com/submit
) Biomed Central 